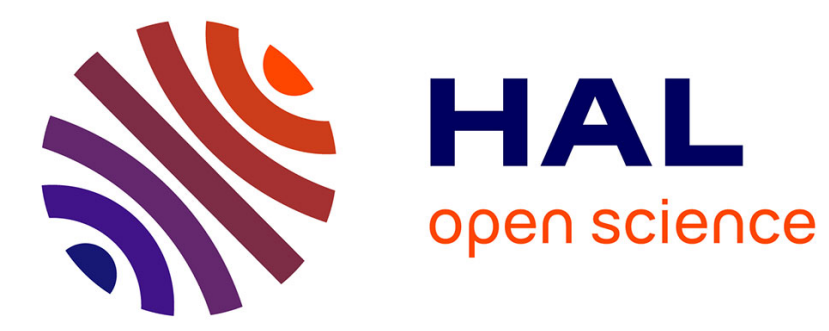

\title{
Fertility in the absence of self-control
}

Bertrand Wigniolle

\section{To cite this version:}

Bertrand Wigniolle. Fertility in the absence of self-control. Mathematical Social Sciences, 2013, pp.71-86. 10.1016/j.mathsocsci.2013.02.001 . halshs-00823264

\section{HAL Id: halshs-00823264 https://shs.hal.science/halshs-00823264}

Submitted on 16 May 2013

HAL is a multi-disciplinary open access archive for the deposit and dissemination of scientific research documents, whether they are published or not. The documents may come from teaching and research institutions in France or abroad, or from public or private research centers.
L'archive ouverte pluridisciplinaire HAL, est destinée au dépôt et à la diffusion de documents scientifiques de niveau recherche, publiés ou non, émanant des établissements d'enseignement et de recherche français ou étrangers, des laboratoires publics ou privés. 


\title{
Fertility in the absence of self-control*
}

\author{
Bertrand Wigniolle ${ }^{\dagger}$
}

March 8, 2013

\begin{abstract}
This paper studies the quantity-quality trade-off model of fertility, under the assumption of hyperbolic discounting. It shows that the lack of self-control may play a different role in a developed economy and in a developing one. In the first case, characterized by a positive investment in quality, the lack of self control may tend to reduce fertility. In the second case, it is possible that the lack of self-control leads to both no investment in quality and a higher fertility rate. It is also proved that if parents cannot commit on their investment in quality, a small change of parameters may lead to a jump in fertility and education.
\end{abstract}

JEL classification: D91, J13, O12

Keywords: endogenous fertility, quasi-hyperbolic discounting.

*I thank two anonymous referees for helpful comments. I also thank participants to Public Economic Theory conference in Seoul (2008), where a preliminary version of this paper was presented, and particularly David de la Croix and Hippolyte d'Albis. I have also benefited from helpful comments at the EQUIPPE seminar in the University of Lille 1.

${ }^{\dagger}$ Paris School of Economics and University of Paris 1. Address: C.E.S., Maison des Sciences Economiques, 106-112, boulevard de l'hôpital, 75647 Paris Cedex 13, France. Tel: +33 (0)1 440781 98. Email : wignioll@univ-paris1.fr. 


\section{Introduction}

From the seminal articles of Becker and Lewis (1973), and Becker and Tomes (1976), the benchmark theory of fertility decisions within the family is the quantity-quality trade-off model. According to this model, the quality and quantity of children are both endogenous variables. Fertility behaviors and investments in children's human capital are consciously and jointly determined by parents. This theory explains fertility and education behaviors as an optimal choice of the household, depending on its income and on the costs of quality and quantity. One major outcome of this literature is to provide an explanation of education and fertility behaviors in developing and developed countries. In developing countries, the cost of quantity is relatively low with respect to the family income, and the cost of quality relatively high. This may explain a high investment in quantity and a low investment in quality in such countries. In developed economies, the cost of quantity is relatively high with respect to the family income, and the cost of quality relatively low. This may result in a low fertility rate and in a high investment in education.

In this paper, I argue that this theory is founded on the implicit assumption of perfect self-control of the household. Indeed, as education decisions are taken after the fertility decision, it is not obvious that the education decision ex-post is consistent with the education decision planned at the time of the fertility choice. This problem of self-control exists if agents are endowed with a non recursive utility function, for instance if the flows of instantaneous utilities are discounted with (quasi)-hyperbolic discounting.

How self-control problems can change the trade-off between quantity and quality? This paper shows that the lack of self-control may play a different role in a developed economy and in a developing one. In a developed country, the lack of self control may tend to reduce fertility and increase education, whereas it may reduce education and increase fertility in a developing country. Moreover, compulsory schooling may play the role of a commitment technology in a developing country that allows to reduce fertility.

Recently, a growing literature has stressed the assumption of (quasi)hyperbolic discount rates. It seems more consistent with laboratory experiments that find a negative relationship between discount rates and time delay (see e. g. Loewenstein, and Thaler (1989)). The consequences of quasihyperbolic discounting have been studied in various frameworks. Many articles have been concerned with savings behavior, mainly Harris and Laibson (2001) and Laibson (1997). Diamond and Köszegi (2003) applied hyperbolic discounting to the early retirement pattern of workers. Barro (1999) introduced this assumption in a standard growth model. Wrede (2009) applies 
quasi-hyperbolic discounting to the timing and number of births, pointing out a possible postponement of births.

A recent article by Salanié and Treich (2006) has made a breakthrough in this literature. In discrete time, quasi-hyperbolic discounting is introduced in the intertemporal utility function of the consumer by adding an extra parameter $\beta \leq 1$ that represents the bias for the present. The instantaneous flows of utility are weighted by the discount factors: $1, \beta \delta, \beta \delta^{2}, \beta \delta^{3}$, etc. The standard assumption of exponential consumers is obtained for $\beta=1$. Hyperbolic consumers have a bias for the present $\beta<1$. In order to evaluate the impact of self-control on behaviors, most articles have compared the results obtained for $\beta<1$ with that obtained for $\beta=1$. The point made by Salanié and Treich is that this comparison is not appropriate to isolate the effect of a lack of self-control, as $\beta$ also modifies the preferences of the consumer. The only pertinent comparison is between the behavior of a consumer with commitment power, and that of a consumer without this power.

In this paper I consider a simple model in which parents arbitrate between the quantity and the quality of their children, as in the benchmark models of Becker and Lewis (1973) and Becker and Tomes (1976). These seminal contributions used a static microeconomic model of demand for quantity and quality of children. In order to study the problem of time consistency of the behaviors, the dynamic structure of the problem must be introduced, here the fact that the choice of fertility is taken before the choice of education. Self-control problems may only occur in a dynamic model that include (at least) three periods, with one decision in the first period and one in the second one.

The household's utility depends on the flows of instantaneous utility obtained during three periods. These flows are discounted with a quasihyperbolic discount factor. In the first period, the household chooses the quantity of children. Each Child entails a cost in time for the household (mainly for the wife) and implies a reduction of income. This cost comes from child rearing and the primary education given inside the family. In the second period, the household chooses the quality level (the education level) given to each child. The education cost is proportional to the number of children and to the level of quality. Finally, in the third period the flow of utility depends positively on both quantity and quality levels. This last assumption corresponds to the altruistic feeling of parents that value both the number and the quality of their children.

The decision process within the household is based on the following assumptions. There is no problem of coordination between spouses in the household, following the unitary model: at each period parents make a joint 
decision or there is a household head that makes all the decisions. But, as preferences are not time-consistent, there is a conflict between the objective functions of the household in periods 1 and 2. From Strotz (1956), the usual way to solve this problem consists in assuming an intrapersonal game. The household is made of a sequence of selves and self $i$ is the decision taker in period $i$. The solution resulting from the equilibrium of this game is called the Temporary Consistent solution (TC in abbreviated form), or the "sophisticated" behavior. This is the solution that is mainly studied in the literature (see, for example, Laibson 1997, Harris and Laibson 2001, or Diamond and Köszegi 2003). Another solution, called the Commitment solution (C in abbreviated form) corresponds to the optimal choice if the first self can take all decisions at all periods. This solution corresponds to a perfect self-control. In practice, the household needs a "Commitment technology" in order to implement the Commitment solution, which means a way to constrain the decision of future selves. For instance, in savings behaviors, the access to an illiquid asset can be viewed as an imperfect commitment technology (see Laibson 1997).

Following Salanié and Treich, the commitment solution for fertility and education is compared to the temporary consistent solution. Two cases are studied. In the first one, interpreted as the case of a developed economy, the cost of quantity is assumed to be relatively high with respect to the family income, and the cost of quality relatively low. In this case, both $\mathrm{C}$ and $\mathrm{TC}$ solutions lead to a positive investment in quality. The impact of the absence of self control depends on the elasticity of substitution of preferences. In the case of an elasticity of substitution greater than one, the absence of self control implies a smaller fertility. The investment in quality is also lower for $\beta$ close to 1 , but higher for a small value of $\beta$.

In the second case, interpreted as the case of a developing economy, the cost of quantity is assumed to be relatively low with respect to the family income, and the cost of quality relatively high. The paper focuses on the situation for which the investment in quality cancels out along the TC solution, whereas it is positive for the C-solution. This case is thinkable for a developing economy ${ }^{1}$, in which children only receive primary education. It leads to a higher fertility rate for the $\mathrm{TC}$ solution than for the $\mathrm{C}$ solution. It means that if the household could commit on its future education investment, it would choose a lower fertility level. For instance, a policy that imposes compulsory attendance at school for children can be viewed as a commitment

\footnotetext{
${ }^{1} \mathrm{~A}$ third case with no investment in quality for both solutions is not studied as it is not interesting. Indeed, for no investment in quality in both solutions, $\mathrm{TC}$ and $\mathrm{C}$ solutions give the same value of fertility.
} 
technology, which is expected to reduce fertility ${ }^{2}$.

The influence of different parameters is considered. The time that parents spent on their children implies a fall in family income, that can be viewed in the model as the (opportunity) cost of fertility $w_{0}$. This opportunity cost mainly corresponds to wife's income. Considering the TC solution and starting from a low value of $w_{0}$, the fertility level is high and no education investment occurs. An increase of $w_{0}$ reduces fertility. At some threshold value, the household begins to invest in quality. At this value, fertility undergoes a jump downward and continues to decrease as $w_{0}$ increases. A second parameter of interest is the cost of education $\tau$. $\tau$ represents the cost of formal education that corresponds to tuition fees, school supplies. It can also incorporate the opportunity cost of child labor for developing economies. Starting from a high value, the economy features a high fertility level with no education investment. As long as no investment in quality occurs, a decrease in $\tau$ has of course no impact on fertility. At some threshold level, the household starts to invest in quality. At this value, fertility undergoes a jump downward and continues to decrease as $\tau$ decreases $^{3}$.

This model offers two novel features with respect to the existing literature. In other words, two characteristics make it difficult to infer directly the impact of self-control on fertility and education from preceding studies of savings, retirement behaviors, etc. The first characteristic is the non-linearity of the budget constraint deriving from the quantity-quality trade-off. The cost of education is the product of quality time quantity. The second characteristic comes from the property that no investment in quality is a possible solution. This solution represents the case of a developing economy, for which no investment in education is provided to children, except primary education.

Concerning the non-linearity of the budget constraint, one consequence is that the lack of self-control may imply lower investment in quality for $\beta$ close to 1 , but higher investment for a small value of $\beta$. This result comes from the property that the cost of quality depends on quantity, and quantity increases with $\beta$. In a model with a linear budget constraint, the lack of self-control would have a monotonic impact.

The second novel feature comes from the case for which no investment in quality is reached along the TC solution. When the quality level chosen by self 2 cancels out, the optimal response for self 1 corresponds to a jump

\footnotetext{
${ }^{2}$ It is possible to consider a more general model with more than 3 periods of life for agents. In this case the lack of commitment implies that children stop earlier their studies in developing economies.

${ }^{3}$ For a high elasticity of substitution in the household preferences, it is possible that the evolution of fertility becomes non-monotonic with $\tau$.
} 
in fertility. In other words, fertility is not continuous at the point for which quality cancels out. This property is interesting, as it means that in the neighborhood of this point, a small change in some parameters can lead to a big change in fertility. For example, a small increase in the opportunity cost of quantity can lead to a big reduction in fertility. This result can be explained considering the objective function of self 1 , along the TC solution. When quality cancels out, the response function of self 2 undergoes a discontinuity of its derivative. Whereas this derivative is negative for a positive investment in quality (quality is a decreasing function of quantity), the derivative is equal to zero when quality cancels out. As there is a discrepancy between the objective functions of selves 1 and 2, the derivative of the self 1 objective function undergoes a jump when quality cancels out. For this reason, two levels of fertility may exist that are local maxima of the objective function of self 1 . If the change of a parameter leads to a jump from one local maximum to the other one, there is a high variation in fertility at this point.

Few studies have been devoted to this property, that a continuous change in some parameter can induce a jump of an endogenous variable, under quasihyperbolic discounting. It can be true in all models in which the decision of a self is subject to a constraint. Laibson (1997) was the first to point out the existence of discontinuous optimal strategies with quasi-hyperbolic discounting, in a model of savings with imperfect capital markets. To avoid the difficulties related to the non-convexity of the problem, he introduced a restriction on the labor income process that ruled out the possibility of corner solutions and discontinuous equilibrium strategies. Harris and Laibson (2002) have provided the most detailed study of this question. They give an intuition of such pathologies. They present the results of numerical simulations, and conclude that such pathologies do not arise when the model is calibrated with empirically sensible parameter values. Wigniolle (2012) remarks that the calibrations in Harris and Laibson that can eliminate the discontinuous strategies (a value of $\beta$ close to 1 , a small value for the elasticity of substitution) are also those that make negligible the impact of hyperbolic discounting. In other words, when hyperbolic discounting matters, it is necessary to deal with such pathologies. He provides a detailed study of such discontinuous strategies in a simple framework that allows a complete characterization.

These different studies point out the role of $\beta$ : if $\beta$ can depart significantly from 1 , the existence of discontinuous strategies may occur. The value of $\beta$ may depend on the time horizon of decisions. If the frequency of decisions is high, a value close to 1 is expected. If the interval of time between two decisions is high, a low value of $\beta$ may be relevant. For decisions concerning fertility and education, it is reasonable to assume a low frequency and a 
small value of $\beta$. Therefore, it seems relevant to expect a strong impact of quasi-hyperbolic discounting on decisions and the occurrence of discontinuous strategies cannot be ignored.

This work can also be compared with the literature about collective choices with non-cooperative behaviors (cf. the seminal contributions of Ulph 1988, Woolley 1988 and Chen and Woolley 2001). This paper follows another way, considering a unitary model of the household: coordination problems within the household do not exist in order to take decisions during a given period; the coordination problem exists in intertemporal choices between two successive selves as preferences are not time-consistent. Some similarities can be found between these two types of models: in both models, non-cooperative behaviors may lead to an equilibrium that is not Pareto-optimal, there may exist under/over provision of a public good within the family. However, there exists an important difference due to the intertemporal structure of the decision process: in the game between selves living at different periods, the self that plays first has a strategic advantage. She/he can play a strategy in taking into account the best response functions of future selves. The resulting Nash equilibrium is not Pareto-optimal in general, and this inefficiency is due to time inconsistency. The optimal policy in this context consists in introducing a commitment technology that allows to implement the commitment solution (C solution). The efficiency of some institutional mechanism to play the role of a commitment technology is then an important topic. In the context of the quantity-quality trade-off model of fertility, compulsory school can be viewed as an example of a commitment technology. Compulsory school attendance implies a minimal investment of parents in the quality of their children. Therefore, it can be viewed by the preceding self as a credible commitment on the future education of children, and it can lead to a fertility choice that is closer to the optimal one.

Another difference between the two types of models is that quasi-hyperbolic discounting may give birth to non-discontinuous strategies. This property was discussed in detail above. For the non-cooperative solution (TC), this may imply a jump of fertility when the investment in education cancels out. This novel feature may have strong economic implications. For instance, there may exist threshold values in a policy promoting education or fertility: this type of policy may have first a smooth effect on behaviors. At some threshold value, a large drop in education and fertility may be observed.

A generalization of both models could be to consider coordination problems both within a given period among spouses and between periods among the selves of each spouse. With such assumptions, two sources of nonoptimality in the decision process would exist. 
Section 2 presents the model. Section 3 gives the fertility decisions for developed and developing economies. Section 4 studies how fertility and education decisions respond to changes in their costs. Section 5 concludes. A final appendix gives the proofs.

\section{The model}

\subsection{Basic assumptions}

The household behavior derives from a utility function defined on three periods and associated with a quasi-hyperbolic discounting factor. ${ }^{4}$ The life-cycle of the household is represented in a very simple way. In the first period, the household chooses the quantity (number) of children. Children entail a cost for rearing and primary education in the family that is assumed to be mainly a cost in time proportional to the number of children. In the second period, the household chooses the quality (education level) of children. Quality results from formal education provided by teachers and it gives rise to a financial cost for parents. Parents choose the same level of quality for each child. In the third period, children become autonomous and their well-being depends on the human capital that they have accumulated when young.

In period 1, self 1 preferences are given by the utility function:

$$
u\left[c_{1}\right]+\beta \delta u\left[c_{2}\right]+\beta \delta^{2} m^{\eta} U\left(q_{0}+q\right)
$$

with

$$
u(x)=\frac{x^{1-\frac{1}{\sigma}}}{1-\frac{1}{\sigma}},
$$

$\eta<1$ and $\sigma>0 . \quad c_{1}$ and $c_{2}$ are respectively the consumption levels of the household in periods 1 and $2 .^{5} \quad \beta$ and $\delta$ are two positive coefficients not greater than $1 . \delta$ is the usual discount parameter and $\beta$ is the bias for the present. $m$ is the number (quantity) of children. $q_{0}+q$ is the quality level of each child that is the sum of an exogenous value $q_{0}$ and of the amount of education $q$ financed by parents. $U\left(q_{0}+q\right)$ is the life-cycle utility level of each child, that is assumed to depend on their human capital. This formulation relies on three assumptions that are often considered in the literature (see e.g. Becker and Barro 1988, Becker et alii 1990 or Jones and Schoonbroodt 2010).

\footnotetext{
${ }^{4}$ An objective function defined on three periods with one decision in the two first periods is the simplest framework in order to study self-control problems.

${ }^{5}$ In the two first periods, children consumption does not appear explicitly. It is assumed to be part of parents' consumption. A simple justification for this assumption is that children consumption may be equal to a constant share of parents' consumption.
} 
First, parents have altruistic feelings to their children: their utility depends on the one of each child. Second, parents likes having children: utility is increasing with the number $m$ of children. Third, this increase is subject to diminishing returns: an increase in $m$ leads to an increase in the utility of parents that is less than proportional.

As $U\left(q_{0}+q\right)$ can be seen as the value function of a child, it is natural to assume that

$$
U(x)=\lambda u(x)
$$

with $\lambda$ some positive constant. To keep a minimum number of parameters of interest, it is possible to choose the units of the different variables in such a way that $\lambda=1 .^{6}$

$\eta$ and $\sigma$ cannot take any value to obtain a concave function that satisfies the three preceding properties. As it is established in Jones and Schoonbroodt, only the following restrictions are admissible:

- either $0<1-1 / \sigma \leq \eta<1$

- or $0>1-1 / \sigma \geq \eta>-1$

To be able to deal with these two cases in the simplest framework, the following assumption is retained: $\eta=1-1 / \sigma$. Under this condition, the two cases correspond to $\sigma>1$ or $1 / 2<\sigma<1$. The third term of the utility function can now be written

$$
\frac{\left[m\left(q_{0}+q\right)\right]^{1-\frac{1}{\sigma}}}{1-\frac{1}{\sigma}}
$$

The assumption $\eta=1-1 / \sigma$ is equivalent to assume that parents value the total revenue of their children, which can also be viewed as a particular warm glow motivation for altruism.

The budget constraint of the family in period 1 is:

$$
c_{1}=w_{1}+w_{0}(1-\phi m)
$$

The family income consists of two parts: a constant part $w_{1}$, and a variable part $w_{0}(1-\phi m)$ that depends on child quantity $m . \phi$ is the time cost for rearing and primary education in the family of one child. $w_{0}$ is the opportunity cost of parents to provide primary education to children. It

\footnotetext{
${ }^{6}$ Our formulation of altruism can also be viewed as a warm glow motivation of parents to give some education to their children, following Andreoni (1989). Such type of formulations have been extensively used by different authors, including Galor and Weil (2000) and De la Croix Doepke (2003) that use the same assumption as this paper.
} 
is an average of the opportunity costs of parents weighted by their relative participation to children rearing, assuming that both spouses devote time to children primary education. $w_{1}$ is the part of household revenues that does not depend on the time devoted to children. As females in general devote more time to children, $w_{0}$ can be viewed approximately as the opportunity cost of time for the wife (wife's income) and $w_{1}$ as husband's income. The resulting consumption level of the household is $w_{1}+w_{0}(1-\phi m)$.

In period 2 , the family income is $w_{2}{ }^{7}$ The education cost of children is now a financial cost, corresponding to the financing of school fees. $\tau$ is the unit cost for one unit of quality for one child. Therefore $\tau m q$ is the cost of providing a quality $q$ to each of the $m$ children, and the resulting second period consumption level of the household is

$$
c_{2}=w_{2}-\tau m q
$$

In period 3 , the $m$ children become autonomous from their parents. Their intertemporal utility depends on their human capital level $\left(q_{0}+q\right)$. Parents are altruistic and value the well-being of their children.

Some remarks can be done on the utility function of self 1 . First, consumption levels of parents do no more depend on spending for their children from period 3. Therefore it is not useful to include parents consumptions in future periods as they will not play any role in the choice of quantity and quality of children. Second, it is assumed that the altruism term in the utility function that depends on quantity and quality of children appears in period 3. It would be possible to introduce other altruism terms in periods 1 and 2 , depending on fertility in period 1 , and on fertility and education in period 2. These terms would only make more complicated the analysis, but they would not add new features to the model. The model is designed in order to remain tractable and our formulation is the simplest one allowing to take into account the quality-quantity trade-off in an intertemporal setting. ${ }^{8}$ Third, it is assumed that parents choose the same level of quality for each child. This assumption can be proved from the concavity of the objective function of the household: it is optimal to give the same education level to each child.

\footnotetext{
${ }^{7}$ In period 2, education only implies a financial cost and the model is unitary. Therefore, all results will only depend on total income $w_{2}$ and not on the income earned by each spouse.

${ }^{8}$ Unlike most of articles that study the marginal effect of a bias for the present, the present paper characterizes cases in which the problem of self-control may induce qualitative changes in the behavior of agents, when quality cancels out. We then need a formulation that allows an explicit expression for agents decisions.
} 
In the literature about fertility decisions, $m$ is usually taken as a continuous variable, since the seminal contributions of Becker and Lewis (1973), and Becker and Tomes (1976). This allows to make standard optimization calculations.

In period 2 , self 2 preferences are given by:

$$
u\left[c_{2}\right]+\beta \delta m^{\eta} u\left(q_{0}+q\right)
$$

The discount factor between period 3 and period 2 is $\delta$ if it is computed by self 1 , and $\beta \delta$ if it is computed by self 2 . The parameter $\beta$ indicates whether there is a self-control problem $(\beta<1)$ or not $(\beta=1)$.

Following Salanié and Treich (2006), the time-consistent solution is compared to the commitment solution. The time-consistent solution (TC) is the non cooperative equilibrium obtained from the game played by selves 1 and 2. More precisely, self 2 chooses $q, m$ being given. Self 1 chooses $m$, taking into account the best response function of self 2 . The commitment solution (C) is obtained by assuming that self 1 can choose both $m$ and $q$.

\subsection{Investment in quality}

\section{The best response function of self 2 for the TC solution}

Self 2 takes $m$ as given and chooses $q$ following his best response function:

$$
q^{T C}(m)=\underset{(q)}{\arg \max }\left\{\begin{array}{c}
u\left[w_{2}-\tau m q\right]+\beta \delta u\left[m\left(q_{0}+q\right)\right] \\
\text { s. t. } q \geq 0
\end{array}\right.
$$

The solution to this program can be interior $(q>0)$ or not. Defining the threshold

$$
\bar{m}^{T C} \equiv \frac{(\beta \delta / \tau)^{\sigma} w_{2}}{q_{0}}
$$

the best response function of self 2 is:

$$
q^{T C}(m)=\left\{\begin{array}{cl}
\frac{(\beta \delta / \tau)^{\sigma} \frac{w_{2}}{m}-q_{0}}{1+(\beta \delta)^{\sigma} \tau^{1-\sigma}} & \text { if } m \leq \bar{m}^{T C} \\
0 & \text { if } m \geq \bar{m}^{T C}
\end{array}\right.
$$

$q^{T C}(m)$ is a non-increasing function of $m$.

\section{The commitment solution}

Assume that self 1 can commit in period 1 on a choice of $q$ in period 2. To compare this solution with the preceding one, it is useful to split the resolution in two steps: firstly the optimal choice of $q$ for $m$ given, secondly 
the optimal value of $m$, in taking into account the effect of $m$ on the optimal choice of $q$. For $m$ given, defining a new threshold

$$
\bar{m}^{C} \equiv \frac{(\delta / \tau)^{\sigma} w_{2}}{q_{0}}
$$

the optimal value of $q$ if self 1 can commit on it in period 1 is:

$$
q^{C}(m)=\left\{\begin{array}{cc}
\frac{(\delta / \tau)^{\sigma} \frac{w_{2}}{m}-q_{0}}{1+\delta^{\sigma} \tau^{1-\sigma}} & \text { if } m \leq \bar{m}^{C} \\
0 & \text { if } m \geq \bar{m}^{C}
\end{array}\right.
$$

$q^{C}(m)$ is a non increasing function of $m$. It is clear that, for $m$ given, $q^{C}(m) \geq q^{T C}(m)$ with a strict inequality when $q^{C}(m)>0$. For a given value of fertility, self $1^{\prime} s$ optimal investment in quality is higher than that chosen by self 2 .

Remark: As usual, the fertility rate is assumed to be a continuous variable. This simplifying assumption leads to meaningless results for $m$ tending toward 0 . Indeed, $q^{C}(m)$ and $q^{T C}(m)$ tend to be infinite when $m$ tends toward 0 , with a discontinuity in $m=0$. Thus, it will be appropriate to eliminate parameter values leading to fertility rates close to 0 .

\section{$3 \quad$ Fertility decisions under quasi-hyperbolic discounting}

This section studies the impact of quasi-hyperbolic discounting on fertility and education decisions. The time-consistent solution is compared to the commitment solution. One important output of the quantity-quality tradeoff model of fertility is to explain fertility and education behaviors through standard economic variables: the costs of quantity and quality of children with respect to family income. In such a framework, the evolution of behaviors through the development process can be explained by the changes in the relative costs. ${ }^{9}$ In a developing economy, the cost of quantity ( $\phi w_{0}$ in our model) is low relatively to the income of the family as women have low opportunities on the labor market. In a developed economy, this relative cost is high as women have more opportunities to participate to the labor market. As for the cost of education $\tau$, it is high with respect to the income of the family in a developing economy, and low in a developed economy. Indeed, developed economies often feature high level of public subsidy in education.

\footnotetext{
${ }^{9} \mathrm{~A}$ general presentation of this literature is given by Birdsall (1988).
} 
As a consequence, the quantity-quality trade-off model of fertility may explain why fertility is high in developing economies and education is low. It may also be the case that children only receive primary education within the family, but no formal education in a school, that corresponds to the case $q=0$ in the model.

Following this analysis, the impact of hyperbolic discounting will be studied in two cases. In the first case, fertility is derived when it is associated with a positive investment in education. In the second case, the corner solution in which education cancels out is studied. The first case may occur when the cost of quantity $\left(\phi w_{0}\right)$ is high enough and the cost of education $\tau$ is low enough with respect to family income. The second case may occur when $\left(\phi w_{0}\right)$ is low and $\tau$ is high with respect to family income. The first case will be referred as the situation of a developed economy, and the second case as the one of a developing country. A complete characterization of these two cases will be provided related to parameter values.

\subsection{The developed economy}

In this part, the case of the developed economy is studied, for which the cost of quantity is assumed to be relatively high with respect to the family income, and the cost of quality relatively low. The time-consistent solution is compared with the commitment solution, when both are interior solutions: $q>0$.

\section{The time-consistent solution}

Along the time-consistent solution, self 1 chooses $m$, taking into account the best response function of self 2 given by equation (4). By assumption, $m$ is such that $q^{T C}(m)>0$ for a developed economy. Self 1's program is:

$$
\begin{aligned}
& \max _{m \geq 0} u\left[w_{1}+w_{0}(1-\phi m)\right]+\beta \delta u\left[w_{2}-\tau m q^{T C}(m)\right]+\beta \delta^{2} u\left[m\left(q_{0}+q^{T C}(m)\right)\right] \\
& \quad \text { Defining }
\end{aligned}
$$

$$
\begin{aligned}
A(\beta) & \equiv \frac{\left(1+\delta^{\sigma} \beta^{\sigma-1} \tau^{1-\sigma}\right)^{\sigma}}{\left(1+\delta^{\sigma} \beta^{\sigma} \tau^{1-\sigma}\right)^{\sigma-1}} \\
B & \equiv\left(\frac{\tau q_{0}}{\phi w_{0}}\right)^{\sigma}
\end{aligned}
$$

the time-consistent solution is:

$$
m^{T C}=\frac{(\beta \delta)^{\sigma} A(\beta) B\left(w_{1}+w_{0}\right)-w_{2}}{\tau q_{0}+\phi w_{0}(\beta \delta)^{\sigma} A(\beta) B}
$$


This solution is valid only if $m^{T C}>0$, which is satisfied if

$$
H(\beta) \equiv(\beta \delta)^{\sigma} A(\beta) B>\frac{w_{2}}{w_{1}+w_{0}}
$$

Following the preceding remark, the parameter values will be restricted in such a way that (9) will hold in what follows.

\section{The commitment solution}

Along the commitment solution, self 1 chooses both $m$ and $q$. This solution can be obtained using equation (5) with $q^{C}(m)>0$ by assumption. The program is:

$$
\max _{m \geq 0} u\left[w_{1}+w_{0}(1-\phi m)\right]+\beta \delta u\left[w_{2}-\tau m q^{C}(m)\right]+\beta \delta^{2} u\left[m\left(q_{0}+q^{C}(m)\right)\right]
$$

The commitment solution is

$$
m^{C}=\frac{(\beta \delta)^{\sigma} A(1) B\left(w_{1}+w_{0}\right)-w_{2}}{\tau q_{0}+\phi w_{0}(\beta \delta)^{\sigma} A(1) B}
$$

This solution is valid only if $m^{C}>0$, which gives the condition

$$
(\beta \delta)^{\sigma} A(1) B>\frac{w_{2}}{w_{1}+w_{0}}
$$

\section{Comparison between TC and C solutions}

The only difference between the two expressions (8) and (10) is the term $A(\beta)$ in place of $A(1)$. As $m$ is increasing with respect to $A, m^{T C}<m^{C}$ if and only if $A(\beta)<A(1)$. It is easy to find:

$$
\frac{d \ln [A(\beta)]}{d \beta}=\frac{\sigma(\sigma-1) \delta^{\sigma} \tau^{1-\sigma} \beta^{\sigma-2}(1-\beta)}{\left(1+\delta^{\sigma} \beta^{\sigma-1} \tau^{1-\sigma}\right)\left(1+\delta^{\sigma} \beta^{\sigma} \tau^{1-\sigma}\right)}
$$

As $\beta<1, A(\beta)<A(1) \Longleftrightarrow \sigma>1$.

If $\sigma>1, m^{T C}<m^{C}$ : the time-consistent solution leads to a lower fertility. As self 2 does not invest enough in education from the point of view of self 1 , and as education choice decreases with fertility, self 1 's best response is a reduction in fertility. If self 2 could commit on a higher level of quality (for instance, if he could commit on the behavior $q^{C}(m)$ ), self 1 would invest more in the quantity of children.

In the opposite case $\sigma<1$, the result is reversed. As self 2 under-invests in quality, self 1 increases quantity with respect to the commitment solution.

This result is close to the one obtained by Salanié and Treich (2006), in a model in which the decision variable of agents is savings. Applying 
their results to a CES utility function (2), they find that the time-consistent solution leads to undersavings iff $\sigma>1$.

In the case $\sigma<1$, the lack of self control leads to higher fertility $m^{T C}>$ $m^{C}$. Therefore, it also leads to a lower quality investment : as $m^{T C}>m^{C}$, $q^{C}\left(m^{C}\right)>q^{C}\left(m^{T C}\right)>q^{T C}\left(m^{T C}\right)$. The absence of commitment implies more quantity and less quality.

In the case $\sigma>1$, it is not so easy to conclude on quality. Indeed, $q^{C}(m)$ and $q^{T C}(m)$ are decreasing functions, with $q^{C}(m)>q^{T C}(m)$ for a given level of fertility $m$. But, as $m^{T C}<m^{C}$, it is not possible yet to conclude if $q^{C}\left(m^{C}\right) \gtrless q^{T C}\left(m^{T C}\right)$. Proposition 1 proves that parents under-invest in quality when $\beta$ is close to 1 , but they over invest for a low value of $\beta$.

The different results are summarized in the following proposition:

Proposition 1 Assuming an interior solution for $m$ and $q$ ( $m$ and $q>0)$,

- In the case $\sigma<1$, the lack of self control leads to higher fertility $m^{T C}>$ $m^{C}$ and lower investment in education $q^{C}>q^{T C}$.

- In the case $\sigma>1$, the lack of self-control leads to lower investments in quantity $m^{T C}<m^{C}$. The investment in quality is also lower for $\beta$ close to one, but higher for a low $\beta$.

Proof. See Appendix 1

Assumption: $\sigma>1$.

The assumption $\sigma>1$ is retained in what follows. It corresponds to the case favored by Salanié and Treich (2006), in which the lack of self-control leads to under-savings. As a consequence of Proposition 1, for $\sigma>1$ and $\beta$ close to 1 , every commitment mechanism on a higher investment in quality increases fertility. For instance, a public policy in favor of commitment such as compulsory schooling will lead to a higher fertility level. But for a low value of $\beta$, there is over investment in quality. The intuition behind this result is that, for a low value of $\beta$, as $m^{T C}$ becomes weak, the cost of quality is very low. This result is due to the non linearity of the cost of education which depends also on quantity. This non linearity is a particular feature of the quantity-quality trade-off model of fertility.

Another consequence of the case $\sigma>1$ is that the constraint (11) is weaker than (9). Therefore, only (9) will be retained.

\section{Existence of an interior solution}

The time-consistent and commitment solutions must satisfy the following inequalities: $0<m^{T C}<\bar{m}^{T C}, 0<m^{C}<\bar{m}^{C}$. As $m^{T C}<m^{C}$, only three inequalities must be considered: $0<m^{T C}, m^{T C}<\bar{m}^{T C}$ and $m^{C}<\bar{m}^{C}$. 
Condition $m^{T C}>0$ is given by (9).

The inequality $m^{T C}<\bar{m}^{T C}$ gives:

$$
Z(\beta)<\frac{w_{2}}{w_{1}+w_{0}}
$$

with $Z$ defined as:

$$
Z(\beta) \equiv \frac{1}{\frac{\phi w_{0} \beta^{\sigma} \delta^{\sigma} \tau^{-\sigma}}{q_{0}}+\frac{\left(\phi w_{0}\right)^{\sigma}}{\left(\tau q_{0}\right)^{\sigma}(\beta \delta)^{\sigma}}\left(\frac{1+\beta^{\sigma} \delta^{\sigma} \tau^{1-\sigma}}{1+\beta^{\sigma-1} \delta^{\sigma} \tau^{1-\sigma}}\right)^{\sigma}}
$$

Finally, the inequality $m^{C}<\bar{m}^{C}$ gives:

$$
G(\beta)<\frac{w_{2}}{w_{1}+w_{0}}
$$

with

$$
G(\beta) \equiv \frac{1}{\frac{\phi w_{0} \delta^{\sigma} \tau^{-\sigma}}{q_{0}}+\frac{\left(\phi w_{0}\right)^{\sigma}}{\left(\tau q_{0}\right)^{\sigma}(\beta \delta)^{\sigma}}}
$$

It is straightforward to see that $G(\beta)<Z(\beta)$. Therefore there remain two necessary conditions for the existence of an interior solution of the household program: (9) and (12).

\subsection{The developing economy}

This part focuses on the case of a developing economy, in which the cost of quantity is assumed to be relatively low with respect to the family income, and the cost of quality relatively high. From these assumptions, the timeconsistent solution may be a corner solution with no investment in quality $\left(q^{T C}=0\right)$. If the commitment solution is also associated with no education investment $\left(q^{C}=0\right)$, it is straightforward to see that the fertility level will be the same for the two solutions $\mathrm{C}$ and TC. Therefore, this case is not interesting as the lack of self-control has no impact on decisions.

More interesting is the case in which the commitment solution is associated with some positive education investment $\left(q^{C}>0\right)$. In this case, the lack of commitment influences education, and thus fertility behaviors.

\section{The time-consistent solution without investment in quality}

Considering the TC behavior in the corner solution with $q^{T C}=0$, the fertility level $m^{T C}$ is given by the first order condition:

$$
-\phi w_{0}\left[w_{1}+w_{0}(1-\phi m)\right]^{-1 / \sigma}+\beta \delta^{2} m^{-1 / \sigma} q_{0}^{1-1 / \sigma}=0
$$


The solution is denoted by $\tilde{m}^{T C}$ and is equal to:

$$
\tilde{m}^{T C}=\frac{\left(\beta \delta^{2}\right)^{\sigma}\left(\phi w_{0}\right)^{-\sigma} q_{0}^{\sigma-1}\left(w_{1}+w_{0}\right)}{1+\left(\beta \delta^{2}\right)^{\sigma}\left(\phi w_{0}\right)^{1-\sigma} q_{0}^{\sigma-1}}
$$

Using (4), condition $\tilde{m}^{T C}>\bar{m}^{T C}$ ensuring that $q^{T C}=0$ gives the following inequality:

$$
\frac{w_{2}}{w_{1}+w_{0}}<D(\beta)
$$

with

$$
D(\beta) \equiv \frac{1}{\frac{\phi w_{0}(\beta \delta)^{\sigma} \tau^{-\sigma}}{q_{0}}+\frac{\left(\phi w_{0}\right)^{\sigma}}{\left(\tau q_{0}\right)^{\sigma}(\delta)^{\sigma}}}
$$

\section{Comparison with the commitment solutions}

By assumption, the commitment solution is associated with some positive education investment $\left(q^{C}>0\right)$. Therefore, $m^{C}$ is still given by $(10)$, and condition (13) must be fulfilled. The comparison between $\tilde{m}^{T C}$ given by (15), and $m^{C}$ given by (10) gives the following result:

Proposition 2 When the lack of self-control leads to no investment in quality for the time-consistent solution and to a positive investment for the commitment solution, the fertility level is higher for the first time-consistent solution: $\tilde{m}^{T C}>m^{C}$.

Proof. From (15) and (10), the inequality $\tilde{m}^{T C}>m^{C}$ is equivalent to

$$
G(\beta)<\frac{w_{2}}{w_{1}+w_{0}}
$$

This condition holds by assumption, as it corresponds to (13), which was obtained in writing the inequality $m^{C}<\bar{m}^{C}$.

This proposition shows that the lack of self-control has a different impact in the developing economy, as it tends to increase fertility. If self 2 could commit on some positive investment in quality, self 1 would invest less in quantity. In a developed country, a policy measure that favors commitment increases fertility. In a developing economy, such a measure will reduce fertility.

How to understand this result? For the TC solution, while $q^{T C}$ remains positive, self 1 gives birth to fewer children in order to obtain more investment in quality by self 2 . But, when $q^{T C}$ cancels out, decreasing fertility has no more impact on quality. The optimal response of self 1 is now to increase his fertility level. 


\section{Conditions for a positive investment in quality}

Considering the TC behavior, two solutions have been found: one interior solution associated with a positive investment in quality and one constrained solution with no investment in quality. The first one must satisfy the condition $Z(\beta)<\frac{w_{2}}{w_{1}+w_{0}}$ and the second one $\frac{w_{2}}{w_{1}+w_{0}}<D(\beta)$. It is easy to check that $Z(\beta)<D(\beta)$. Therefore, three cases may exist. If $\frac{w_{2}}{w_{1}+w_{0}}>D(\beta)$, only the interior solution exists. If $\frac{w_{2}}{w_{1}+w_{0}}<Z(\beta)$ only the constrained solution exists. If $Z(\beta)<\frac{w_{2}}{w_{1}+w_{0}}<D(\beta)$, the problem is to choose between the two solutions.

To understand this point, it is useful to consider the first order condition of the program of self 1 . Self 1 chooses the optimal value of $m$, taking into account the best response function of self $2 q^{T C}(m)$ :

$$
\begin{aligned}
0= & -\phi w_{0} u^{\prime}\left[w_{1}+w_{0}(1-\phi m)\right]-\tau q^{T C}(m) \beta \delta u^{\prime}\left[w_{2}-\tau m q^{T C}(m)\right] \\
& +\beta \delta^{2}\left(q_{0}+q^{T C}(m)\right) u^{\prime}\left[m\left(q_{0}+q^{T C}(m)\right)\right] \\
& +\beta \delta m \frac{d q^{T C}(m)}{d m}\left\{-\tau u^{\prime}\left[w_{2}-\tau m q^{T C}(m)\right]+\delta u^{\prime}\left[m\left(q_{0}+q^{T C}(m)\right)\right]\right\}
\end{aligned}
$$

For the commitment solution, the first order condition is the same, except that $q^{T C}(m)$ is replaced by $q^{C}(m)$. But, for the commitment solution, the expression $-\tau u^{\prime}\left[w_{2}-\tau m q^{C}(m)\right]+\delta u^{\prime}\left[m\left(q_{0}+q^{C}(m)\right)\right]$ cancels out by definition of $q^{C}(m)$. For the time-consistent solution, the expression

$-\tau u^{\prime}\left[w_{2}-\tau m q^{T C}(m)\right]+\delta u^{\prime}\left[m\left(q_{0}+q^{T C}(m)\right)\right]$ is positive, as $q^{T C}(m)$ is implicitly defined by $-\tau u^{\prime}\left[w_{2}-\tau m q^{T C}(m)\right]+\beta \delta u^{\prime}\left[m\left(q_{0}+q^{T C}(m)\right)\right]=0$. This is the consequence of the discrepancy between the objective functions of self 1 and self 2 . For the derivative $d q^{T C}(m) / d m$, there is a discontinuity in $\bar{m}^{T C}$ : this derivative is negative to the left of $\bar{m}^{T C}$, and is zero to the right.

The consequence of this analysis is that the derivative of self 1's objective function is always continuous for the commitment solution. But, for the time-consistent solution, the derivative of self 1's objective function is discontinuous at the point $\bar{m}^{T C}$, with a higher value to the right of $\bar{m}^{T C}$. It is then possible that self 1 objective function admits two local maxima. The function is concave on each interval $\left(0, \bar{m}^{T C}\right)$ and $\left(\bar{m}^{T C},+\infty\right)$ and continuous, but the derivative is discontinuous in $\bar{m}^{T C}$.

Figure 1 presents a numerical simulation with the following values of parameters: $\sigma=2, \tau=0.5, \phi=0.17, \beta=0.5, \delta=1, w_{2}=2, q_{0}=0.5$ and $w_{1}=1$. The different curves are obtained for different values of the parameter $w_{0}{ }^{10}$. The objective function of self 1 with respect to $m$ is drawn. The value

\footnotetext{
${ }^{10}$ The same type of analysis could be carried out with respect to another parameter than $w_{0}$.
} 
$w_{0}=0.951856$ is such that the two local maxima give the same value to the utility. For $w_{0}$ smaller than this value, the optimal behavior is to give birth to many children $\left(\tilde{m}^{T C}\right)$ and to not invest in their education. For $w_{0}$ higher than this value, the optimal behavior is to have a small number of children $\left(m^{T C}\right)$ and to invest in their quality.

Considering self 1's objective function, under the condition $Z(\beta)<\frac{w_{2}}{w_{1}+w_{0}}<$ $D(\beta)$, this function of $m$ has two local maxima: one associated with a positive investment in education $\left(m^{T C}\right)$ and one for which education cancels out $\left(\tilde{m}^{T C}\right)$. Therefore it is necessary to compare the utility levels obtained for each local maximum. $U^{T C}$ denotes the indirect utility level when $q^{T C}$ is positive and $\tilde{U}^{T C}$ the utility level when $q^{T C}$ is zero. The following lemma shows that the equality $U^{T C}=\tilde{U}^{T C}$ implicitly defines a function $V(\beta)$ such that $\frac{w_{2}}{w_{1}+w_{0}}=V(\beta) \Leftrightarrow U^{T C}=\tilde{U}^{T C}$. Moreover, $U^{T C}>\tilde{U}^{T C} \Leftrightarrow \frac{w_{2}}{w_{1}+w_{0}}>V(\beta)$.

Lemma 1 Assume that $Z(\beta)<\frac{w_{2}}{w_{1}+w_{0}}<D(\beta)$. The condition $U^{T C}>\tilde{U}^{T C}$ holds iff:

$$
\begin{aligned}
& \left(\frac{w_{2}}{w_{1}+w_{0}} \frac{\phi w_{0}}{\tau q_{0}}+1\right)^{1-1 / \sigma}\left[1+\left(\frac{\phi w_{0}}{\tau q_{0}}\right)^{1-\sigma}(\beta \delta)^{\sigma} A(\beta)\right]^{1 / \sigma} \\
> & {\left[1+\left(\frac{\phi w_{0}}{q_{0}}\right)^{1-\sigma}\left(\beta \delta^{2}\right)^{\sigma}\right]^{1 / \sigma}+\beta \delta\left(\frac{w_{2}}{w_{1}+w_{0}}\right)^{1-1 / \sigma} }
\end{aligned}
$$

This inequality implicitly defines a function $V(\beta)$ such that

$$
U^{T C}>\tilde{U}^{T C} \Leftrightarrow \frac{w_{2}}{w_{1}+w_{0}}>V(\beta),
$$

and this function satisfies:

$$
Z(\beta)<V(\beta)<D(\beta)
$$

Proof. See Appendix 2.

This lemma allows characterizing the optimal solution for $\frac{w_{2}}{w_{1}+w_{0}} \in[Z(\beta), D(\beta)]$. If $\frac{w_{2}}{w_{1}+w_{0}}>V(\beta)$, the optimal TC-solution is such that $q^{T C}>0$. If $\frac{w_{2}}{w_{1}+w_{0}}<$ $V(\beta)$, the optimal TC-solution is such that $q^{T C}=0$.

\subsection{Existence of the different regimes}

This part provides a characterization of the existence of the different regimes with respect to the parameter $\beta$. It is based on a technical lemma: 
Lemma 2 - $H(\beta)$ is an increasing function of $\beta$, and when $\beta$ goes from 0 to $1, H(\beta)$ goes from 0 to $\left(\delta \tau q_{0}\right)^{\sigma}\left(\phi w_{0}\right)^{-\sigma}\left(1+\delta^{\sigma} \tau^{1-\sigma}\right)>D(1)$. Moreover, for every $\beta, H(\beta)>Z(\beta)$.

- $G, Z, V$ and $D$ are such that: $\forall \beta \in(0,1)$,

$$
G(\beta)<Z(\beta)<V(\beta)<D(\beta)
$$

and

$$
G(1)=Z(1)=V(1)=D(1)=\frac{\left(\delta \tau q_{0}\right)^{\sigma}\left(\phi w_{0}\right)^{-\sigma}}{1+q_{0}^{\sigma-1} \delta^{2 \sigma}\left(\phi w_{0}\right)^{1-\sigma}}
$$

- $G$ increases with $\beta$ and $D$ decreases with $\beta$.

Proof. The proof results from straightforward calculations.

This lemma allows a complete characterization of the different cases. Parameters are restricted to be such that (9) holds, or $w_{2} /\left(w_{1}+w_{0}\right)<H(\beta)$. In this zone, the preceding analysis has shown that the functions $G(\beta)$ and $V(\beta)$ are the pertinent frontiers. The set of parameters can be divided into 3 sub-zones $A, B$ and $C$. The following proposition gives for each zone the corresponding expressions of fertility and education. A numerical illustration (see Figure 2) is provided for the following values of parameters : $\sigma=2$, $\tau=0.5, \phi=0.17, \delta=1, q_{0}=0.5$.

Proposition 3 - The plan $\left(\beta, w_{2} /\left(w_{1}+w_{0}\right)\right)$ can be separated into three zones:

Zone $A=\left\{\left(\beta, w_{2} /\left(w_{1}+w_{0}\right)\right), w_{2} /\left(w_{1}+w_{0}\right)<G(\beta)\right\}$,

Zone $B=\left\{\left(\beta, w_{2} /\left(w_{1}+w_{0}\right)\right), G(\beta)<w_{2} /\left(w_{1}+w_{0}\right)<V(\beta)\right\}$,

Zone $C=\left\{\left(\beta, w_{2} /\left(w_{1}+w_{0}\right)\right), V(\beta)<w_{2} /\left(w_{1}+w_{0}\right)\right\}$.

- Assuming that parameters are such that $w_{2} /\left(w_{1}+w_{0}\right)<H(\beta)$,

in zone $A, q^{C}=q^{T C}=0$ and $\tilde{m}^{C}=\tilde{m}^{T C}$,

in zone $B, q^{T C}=0, q^{C}>0$ and $\tilde{m}^{T C}>m^{C}$,

in zone $C, q^{T C}>0, q^{C}>0$ and $m^{T C}<m^{C}$.

Proof. The proof is a direct consequence of lemmas (1) and (2). 
In zone $A$, the optimal behavior in both cases leads to no investment in quality. When investment in quality cancels out, both solutions are associated with the same level of fertility.

In zone $B$, the time consistent solution leads to a higher level of fertility than the commitment solution, and to no investment in children's quality. If self 2 could commit on a higher investment in education, self 1 would invest less in quantity.

Zone $C$ corresponds to the developed economy with a positive investment in quality. The temporary consistent solution leads to lower investment in quantity. If self 2 could commit on a higher investment in education, self 1 would invest more in quantity.

For a given value of $w_{2} /\left(w_{1}+w_{0}\right)$, it is possible that all three zones $A, B$ and $C$ are successively reached depending on the value of $\beta$.

Two cases may happen. In the case $w_{2} /\left(w_{1}+w_{0}\right)<G(1)$, zone $A$ appears for $\beta$ close to 1 ; zone $B$ for $\beta$ such that $G(\beta)<w_{2} /\left(w_{1}+w_{0}\right)<V(\beta)$; zone $C$ appears only if there exist values of $\beta$ such that $V(\beta)<w_{2} /\left(w_{1}+w_{0}\right)<H(\beta)$. In the case $w_{2} /\left(w_{1}+w_{0}\right)>G(1)$, only zones $B$ and $C$ may exist because $G(\beta)$ is always smaller than $G(1)<w_{2} /\left(w_{1}+w_{0}\right)$. These results show that the impact of $\beta$ on the investments in quality can be ambiguous for the TC solution. Indeed, an increase of $\beta$ has a twofold effect. For a given level of fertility, increasing $\beta$ rises the investment in quality. But an increase in $\beta$ also rises the investment in quantity, which has a negative impact on the investment in quality.

\section{Impact of the costs of fertility and educa- tion}

This section studies how fertility and education behaviors respond to changes of $w_{0}$ and $\tau$.

\subsection{Effect of $w_{0}$}

$w_{0}$ play a crucial role in education and fertility. An increase of $w_{0}$ has a twofold impact: first it increases the opportunity cost of the quantity of children; second, for a given level of fertility, it increases the first period income of the family. The first effect (effect on the price) is expected to dominate the second one (effect on the revenue), as in the standard trade-off model between consumption and leisure. Therefore, the increase of $w_{0}$ is expected to imply a fall in fertility. 
In writing equation (8) under the form:

$$
m^{T C}=\frac{(\beta \delta)^{\sigma} A(\beta)\left(\tau q_{0}\right)^{\sigma} \frac{\left(w_{1}+w_{0}\right)}{\phi w_{0}}-w_{2}\left(\phi w_{0}\right)^{\sigma-1}}{\tau q_{0}\left(\phi w_{0}\right)^{\sigma-1}+(\beta \delta)^{\sigma} A(\beta)\left(\tau q_{0}\right)^{\sigma}}
$$

it is straightforward that $m^{T C}$ is a decreasing function of $w_{0}$ as the numerator is decreasing and the denominator is increasing. Using the same argument, $m^{C}$ given by (10) is also decreasing with respect to $w_{0}$. Finally, when education cancels out, equation (15) can be written again as

$$
\tilde{m}^{T C}=\frac{\left(\beta \delta^{2}\right)^{\sigma} q_{0}^{\sigma-1} \frac{\left(w_{1}+w_{0}\right)}{\phi w_{0}}}{\left(\phi w_{0}\right)^{\sigma-1}+\left(\beta \delta^{2}\right)^{\sigma} q_{0}^{\sigma-1}}
$$

which is decreasing with $w_{0}$.

In all cases, fertility decreases with respect to $w_{0}$. A change of $w_{0}$ can also result in a change of regime, and a drop in fertility. Starting from the fertility level without education $\tilde{m}^{T C}$, an increase of $w_{0}$ implies a decrease in fertility. This change of $w_{0}$ may induce such a decrease in fertility that it becomes optimal to invest in quality. At this point, there is a discontinuity in fertility that experiences a fall between $\tilde{m}^{T C}$ and $m^{T C}$. In the neighborhood of the frontier value of $w_{0}$, a small increase of $w_{0}$ induces a great drop in fertility. This jump is the consequence of the discrepancy between the objective functions of self 1 and self 2 . Figure 3 gives a numerical illustration for the following values of parameters : $\sigma=2, \tau=0.5, \phi=0.17, \beta=0.5, \delta=1$, $w_{2}=2, q_{0}=0.5, w_{1}=1$. Parameters are such that for $w_{0}=0.951856$ there is the discontinuity in fertility.

The frontiers between the different regimes can be characterized with respect to $w_{0}$. They cannot be deduced from Figure 2, as the different functions $H, V$ and $G$ depend on $w_{0}$. The characterization is made in the plan $\left(w_{0}, w_{1}\right)$. As before, parameters are constrained in such a way that $m^{T C}>0$, which corresponds to condition (9). This constraint defines in the plan $\left(w_{0}, w_{1}\right)$ a zone such that $w_{1}>W^{H}\left(w_{0}\right)$, with $W^{H}$ a function defined in Appendix (3). The same method is used for condition (13): a function $W^{G}\left(w_{0}\right)$ is introduced, such that the condition holds iff $w_{1}<W^{G}\left(w_{0}\right)$. Finally, the function $W^{V}\left(w_{0}\right)$ is introduced, such that condition (19) holds iff $w_{1}<W^{V}\left(w_{0}\right)$. The three functions $W^{H}\left(w_{0}\right), W^{G}\left(w_{0}\right)$ and $W^{V}\left(w_{0}\right)$ allow to obtain a characterization of the different regimes in the plan $\left(w_{0}, w_{1}\right)$. This characterization is equivalent to the one given in section 3.3 in the plan $\left(\beta, \frac{w_{2}}{w_{1}+w_{0}}\right)$, but it shows the role of $w_{0}$ in the existence of the different regime.

The following proposition gives the complete characterizations of the different regimes in the plan $\left(w_{0}, w_{1}\right)$, using the frontiers defined by the three functions $W^{H}\left(w_{0}\right), W^{G}\left(w_{0}\right)$ and $W^{V}\left(w_{0}\right)$. 
Proposition 4 - It is possible to define three functions $W^{H}\left(w_{0}\right), W^{G}\left(w_{0}\right)$ and $W^{V}\left(w_{0}\right)$ that are non-decreasing functions of $w_{0}$, and for all $w_{0}$, $W^{G}\left(w_{0}\right)>W^{V}\left(w_{0}\right)$.

- The plan $\left(w_{0}, w_{1}\right)$ can be separated in three zones:

Zone $A=\left\{\left(w_{0}, w_{1}\right), w_{1}>W^{G}\left(w_{0}\right)\right\}$,

Zone $B=\left\{\left(w_{0}, w_{1}\right), W^{V}\left(w_{0}\right)<w_{1}<W^{G}\left(w_{0}\right)\right\}$,

Zone $C=\left\{\left(w_{0}, w_{1}\right), w_{1}<W^{V}\left(w_{0}\right)\right\}$.

- Assuming that parameters are such that $w_{1}>W^{H}\left(w_{0}\right)$,

in zone $A, q^{C}=q^{T C}=0$ and $\tilde{m}^{C}=\tilde{m}^{T C}$,

in zone $B, q^{T C}=0, q^{C}>0$ and $\tilde{m}^{T C}>m^{C}$,

in zone $C, q^{T C}>0, q^{C}>0$ and $m^{T C}<m^{C}$.

Proof. See Appendix 3.

Zone $A$ is obtained for a low value of $w_{0}, q^{C}=q^{T C}=0$ and $\tilde{m}^{C}=\tilde{m}^{T C}$. As the opportunity cost of children is small, fertility is high and parents do not invest in quality.

In zone $B, q^{T C}=0$ but $q^{C}>0$ and $\tilde{m}^{T C}>m^{C}$. For an intermediate value of $w_{0}$, the TC behavior leads to no investment in quality, whereas parents invest in quality along the commitment solution. Fertility is lower for the commitment solution.

In zone $C, q^{T C}$ and $q^{C}$ are both positive and $m^{T C}<m^{C}$. For a high value of $w_{0}$, the TC and $\mathrm{C}$ solutions are associated with a positive investment in quality, and fertility is higher for the commitment solution.

A consequence of these results for the TC behavior is that fertility experiences a strong discontinuity for $w_{0}=\left(W^{V}\right)^{-1}\left(w_{1}\right) \equiv w_{0}^{l}$. In the neighborhood of this value $w_{0}^{l}$, a small increase of $w_{0}$ leads to a large drop in fertility.

Figure 4 shows a numerical simulation of the different zones in the plan $\left(w_{0}, w_{1}\right)$, for the same values of parameters as Figure 3 . For $w_{1}=1$, fertility experiences a discontinuity at the value $w_{0}=0.951856$.

The discontinuity in the optimal strategy of self 1 is a particular feature of the model with quasi-hyperbolic discounting. In the model with exponential discounting, a change in the value of some parameter results in a continuous effect on the choices of the agent. In the model with quasi-hyperbolic discounting, it is possible to observe jumps that are related to the non-concavity 
of self one's objective. This property introduces a qualitative difference in the two models that may have important empirical consequences. If the model with quasi-hyperbolic discounting is relevant, fertility behaviors may undergo large changes for some critical values of the parameters. This may have consequences for the empirical analysis of fertility and for the dynamics of demographic transitions.

\section{2 $\quad$ Effect of $\tau$}

The parameter $\tau$ is the cost of education. An increase of $\tau$ changes the optimal trade-off between quality and quantity. The following proposition summarizes the effect of $\tau$ on fertility and education.

Proposition $5 \quad-m^{C}$ increases when the cost of education $\tau$ increases, and $q^{C}$ decreases.

- If $\sigma$ is small enough, i.e. $\sigma \leq 1 /(1-\beta), m^{T C}$ increases when the cost of education $\tau$ increases, and $q^{T C}\left(m^{T C}\right)$ decreases. If $\sigma>1 /(1-\beta)$, $m^{T C}$ increases with $\tau$ when $\tau$ is small ( $\tau$ in a neighborhood of 0 ). But, numerical simulations show that $m^{T C}$ can be a non monotonic function of $\tau$ (see Figure 6).

- There exists a threshold $\bar{\sigma}$, with $\bar{\sigma}>1 /(1-\beta)$, such that if $\sigma<\bar{\sigma}$, $q^{T C}\left(m^{T C}\right)$ decreases with $\tau$.

Proof. See Appendix 4.

For the commitment solution, an increase of $\tau$ reduces the investment in education, and increases fertility. This result is standard in the basic model of quantity-quality trade-off. For a given level of fertility, an increase of $\tau$ reduces the investment in education $q$. As $q$ is lower, the cost of fertility decreases and fertility increases. For the TC-solution, the same effect is obtained for a small value of $\sigma$. But if $\sigma$ is very high, $\tau$ can have a non monotonic effect.

As for $w_{0}$, there exists a threshold level $\tau^{l}$ such that education cancels out for $\tau>\tau^{l}$. At this point $\tau^{l}$, fertility undergoes a large drop to a higher value, as education falls to zero. Figures 5 and 6 show how fertility may evolve with respect to $\tau$. Figure 5 uses the preceding values for the different parameters: $\sigma=2, \phi=0.17, \beta=0.5, \delta=1, q_{0}=0.5, w_{0}=1, w_{1}=1, w_{2}=2$. The threshold level from which education cancels out is $\tau^{l}=0.516066$.

Figure 6 is an example of parameters leading to a non-monotonic evolution of fertility for a high value of $\sigma . \sigma=4>1 /(1-\beta)=2, \phi=0.28$, 
$\beta=0.5, \delta=1, q_{0}=1.5, w_{0}=1, w_{1}=1, w_{2}=2$. The threshold level is $\tau^{l}=0.341284 . \sigma$ can be interpreted as the elasticity of substitution between consumptions. The value of $\sigma$ is a controversial question in economics. A value close to one corresponds to the one retained in calibrated macroeconomic models. Microeconomic estimations often conclude to a smaller value. A value $\sigma>1 /(1-\beta)$ does not seem to be the most relevant assumption. Therefore, a non monotonic evolution of $m$ with respect to $\tau$ is not very plausible.

An increase of $\tau$ also influences the existence of the different regimes. This question is studied in the plan $\left(\beta, w_{2} /\left(w_{1}+w_{0}\right)\right)$, considering how the different frontiers $G(\beta)$ and $V(\beta)$ are modified. For $\beta$ given, it is straightforward from (14) that $G(\beta)$ in an increasing function of $\tau$. As could be expected, the region $A$ in which no education occurs increases with $\tau$. Consequently there is less space for regions $B$ and $C$. The frontier between regions $B$ and $C$ is defined with the function $V(\beta)$. Appendix 5 shows that $V(\beta)$ increases with $\tau$. A numerical experiment is provided in Figure 7, with the following parameters $\sigma=2, \phi=0.17, \delta=1, q_{0}=0.5, \tau=0.5$ and $\tau=0.7$. The case $\tau=0.7$ is presented with bold lines.

\section{Conclusion}

This paper has studied the quantity-quality fertility model under the assumption of quasi-hyperbolic discounting. The impact of the absence of self control is isolated through the comparison between the TC solution (sophisticated behavior) and the $\mathrm{C}$ solution (commitment solution). The lack of self control may have different impact on fertility in a developed economy and in a developing one. In a developed economy characterized by a positive investment in quality, the lack of self control tends to reduce fertility. In a developing economy, the lack of self-control may lead to both no investment in quality and a higher fertility rate. It is also proved that if parents cannot commit on their investment in quality, a small change of parameters may lead to a jump in fertility and education.

This paper could be extended in different directions. First, the robustness of the results could be studied if the model was enriched by additional assumptions: access to capital markets for the households, imperfect capital markets through borrowing constraints, collective choice within the household, etc. Secondly, a technical improvement could be made by introducing more than three periods and more than two decisions. 


\section{References}

[1] Andreoni, J. (1989). Giving with impure altruism: aplications to charity and Ricardian equivalence. Journal of Political Economy, 97, 1447-1458.

[2] Barro, R. J. (1999). Ramsey meets Laibson in the neoclassical growth model. The Quarterly Journal of Economics, 114(4), 1125-1152.

[3] Becker, G. S. \& Lewis, H. G. (1973). Interaction between quantity and quality in children. Journal of Political Economy, 81, S279-S288.

[4] Becker, G. S. \& Tomes, N. (1976). Child endowments and the quantity and quality of children. Journal of Political Economy, 84, S143-S162.

[5] Becker, G. S. \& Barro, R. J. (1988). A reformulation of the economic theory of fertility. The Quarterly Journal of Economics, 103, 1-25.

[6] Becker, G. S., Murphy, K. M. \& Tamura, R. (1990). Human capital, fertility, and economic growth. Journal of Political Economy, 98, S1237.

[7] Birdsall, N. (1988). Economic approaches to population growth. In H. Chenery, \& T.N. Srinivasan (Eds.), Handbook of Development Economics (pp 477-542). Amsterdam: North-Holland.

[8] Chen, Z. \& Woolley, F. (2001). A Cournot-Nash model of family decision making. Economic Journal, 111, 722-48.

[9] De la Croix, D. \& Doepke, M. (2003). Inequality and growth: why differential fertility matters. American Economic Review, 93, 1091-1113.

[10] Diamond, P. \& Koszegi, B. (2003). Quasi-hyperbolic discounting and retirement. Journal of Public Economics, 87, 1839-1872.

[11] Galor, O. \& Weil, D. N. (2000). Population, technology, and growth: from Malthusian stagnation to the demographic transition and beyond. American Economic Review, 90, 806-828.

[12] Harris, C. \& Laibson, D. (2001). Dynamic choices of hyperbolic consumers. Econometrica, 69, 935-57.

[13] Harris, C. \& Laibson, D. (2002). Hyperbolic discounting and consumption. In M. Dewatripont, L. P. Hansen, \& S. Turnovsky (Eds.), Advances in economics and econometrics: theory and applications (pp 258-298). Cambridge: Cambridge University Press. 
[14] Jones, L. E. \& Schoonbroodt, A. (2010). Complements versus substitutes and trends in fertility choice in dynastic models. International Economic Review, 51, 671-699.

[15] Laibson, D. (1997). Golden eggs and hyperbolic discounting. The Quarterly Journal of Economics, 112, 443-77.

[16] Loewenstein, G. \& Thaler, R. H. (1989). Intertemporal choice. Journal of Economic Perspectives, 3, 181-93.

[17] Salanié, F. \& Treich, N. (2006). Over-savings and hyperbolic discounting. European Economic Review, 50, 1557-1570.

[18] Strotz, R. H. (1956). Myopia and inconsistency in dynamic utility maximization. Review of Economic Studies, 23, 165- 80.

[19] Ulph, D. (1988). A general noncooperative Nash model of household behaviour. mimeo, University of Bristol.

[20] Wrede M. (2011). Hyperbolic discounting and fertility. Journal of Population Economics, 24, 1053-1070.

[21] Wigniolle B. (2012). Savings behavior with imperfect capital markets: when hyperbolic discounting leads to discontinuous strategies. Economics Letters, 116, 186-189.

[22] Woolley, F. R. (1988). A non-cooperative model of family decision making. TIDI Working Paper 125, London School of Economics.

\section{Appendixes}

\subsection{Appendix 1}

The comparison between $m^{T C}$ and $m^{C}$ is made in the text. In the case $\sigma<1$, the comparison between $q^{C}\left(m^{C}\right)$ and $q^{T C}\left(m^{T C}\right)$ is simple and is made in the text. It remains to compare $q^{C}\left(m^{C}\right)$ and $q^{T C}\left(m^{T C}\right)$ when $\sigma>1$.

First, it appears that:

$$
q^{T C}\left(m^{T C}\right)+q_{o}=\frac{(\beta \delta / \tau)^{\sigma}\left(\frac{w_{2}}{m^{T C}}+\tau q_{0}\right)}{1+(\beta \delta)^{\sigma} \tau^{1-\sigma}}
$$


and

$$
q^{C}\left(m^{C}\right)+q_{o}=\frac{(\delta / \tau)^{\sigma}\left(\frac{w_{2}}{m^{C}}+\tau q_{0}\right)}{1+(\delta)^{\sigma} \tau^{1-\sigma}}
$$

From (8), is obtained:

$$
\frac{w_{2}}{m^{T C}}+\tau q_{0}=\frac{(\beta \delta)^{\sigma} A(\beta) B\left[\tau q_{0}\left(w_{1}+w_{0}\right)+w_{2} \phi w_{0}\right]}{(\beta \delta)^{\sigma} A(\beta) B\left(w_{1}+w_{0}\right)-w_{2}}
$$

From (10), is obtained:

$$
\frac{w_{2}}{m^{C}}+\tau q_{0}=\frac{(\beta \delta)^{\sigma} A(1) B\left[\tau q_{0}\left(w_{1}+w_{0}\right)+w_{2} \phi w_{0}\right]}{(\beta \delta)^{\sigma} A(1) B\left(w_{1}+w_{0}\right)-w_{2}}
$$

As $A(1)=1+\delta^{\sigma} \tau^{1-\sigma}$, it follows:

$$
\begin{gathered}
q^{T C}\left(m^{T C}\right)+q_{o}<q^{C}\left(m^{C}\right)+q_{o} \Leftrightarrow \\
\frac{(\beta)^{\sigma}}{1+(\beta \delta)^{\sigma} \tau^{1-\sigma}} \frac{A(\beta)}{(\beta \delta)^{\sigma} A(\beta) B\left(w_{1}+w_{0}\right)-w_{2}}<\frac{1}{(\beta \delta)^{\sigma} A(1) B\left(w_{1}+w_{0}\right)-w_{2}}
\end{gathered}
$$

After rearranging and using the expression (6) of $A(\beta)$, it is possible to write this inequality:

$$
0<B\left(w_{1}+w_{0}\right) \delta^{\sigma}-w_{2} f(\beta)
$$

with

$$
f(\beta) \equiv \frac{\left(\frac{1+\delta^{\sigma} \beta^{\sigma} \tau^{1-\sigma}}{\beta+\delta^{\sigma} \beta^{\sigma} \tau^{1-\sigma}}\right)^{\sigma}-1}{1-\beta^{\sigma}}
$$

Firstly the inequality (23) is studied in a neighborhood of $\beta=1$. In setting $x=\beta^{\sigma}$, a function $g$ is introduced such that:

$$
g(x) \equiv \frac{\left(\frac{1+x \delta^{\sigma} \tau^{1-\sigma}}{x^{1 / \sigma}+x \delta^{\sigma} \tau^{1-\sigma}}\right)^{\sigma}-1}{1-x}=f(\beta)
$$

The limit of $g$ when $x$ tends toward 1 is equal to the limit of $f$ in $\beta=1$. Defining a function $h(x)$ such that:

$$
h(x) \equiv\left(\frac{1+x \delta^{\sigma} \tau^{1-\sigma}}{x^{1 / \sigma}+x \delta^{\sigma} \tau^{1-\sigma}}\right)^{\sigma}
$$

this limit is equal to $-h^{\prime}(1)$. Taking the derivative of the logarithm of $h$ in $x=1$, it is obtained:

$$
h^{\prime}(1)=h^{\prime}(1) / h(1)=\frac{\sigma \delta^{\sigma} \tau^{1-\sigma}}{1+\delta^{\sigma} \tau^{1-\sigma}}-\frac{1+\sigma \delta^{\sigma} \tau^{1-\sigma}}{1+\delta^{\sigma} \tau^{1-\sigma}}=-\frac{1}{1+\delta^{\sigma} \tau^{1-\sigma}}
$$


Thus, with $\beta=1$, (23) becomes:

$$
0<B\left(w_{1}+w_{0}\right) \delta^{\sigma}-\frac{w_{2}}{1+\delta^{\sigma} \tau^{1-\sigma}}
$$

which is satisfied as it corresponds to (11) with $\beta=1$. It is then proved that $q^{T C}<q^{C}$ in a neighborhood of $\beta=1$.

Secondly, the inequality (23) is studied for a low value of $\beta$. When $\beta$ tends toward $0, f(\beta)$ tends to be infinite, the inequality (23) cannot be satisfied, and $q^{T C}>q^{C}$. $\beta$ close to 0 is not possible as it implies negative values for $m^{T C}$ and $m^{C}$. The smallest possible value of $\beta$ corresponds to the constraint (9) ensuring $m^{T C}>0$. When $\beta$ tends to this value, the left-hand side of (22) tends to be infinite. Thus, when $\beta$ is low enough, (23) cannot be satisfied, and $q^{T C}>q^{C}$.

\subsection{Appendix 2}

In this appendix, a new notation $x$ is introduced for the expression $\frac{w_{2}}{w_{1}+w_{0}}$. Assume that $Z(\beta)<x<D(\beta)$. The equality $U^{T C}=\tilde{U}^{T C}$ implicitly defines $x$ as a function of $\beta$ : $f(x, \beta)=0$ with

$$
\begin{aligned}
f(x, \beta) \equiv & \frac{\sigma-1}{\sigma}\left(U^{T C}-\tilde{U}^{T C}\right) \frac{1}{\left(w_{1}+w_{0}\right)^{1-1 / \sigma}}= \\
& \left(x \frac{\phi w_{0}}{\tau q_{0}}+1\right)^{1-1 / \sigma}\left[1+\left(\frac{\phi w_{0}}{\tau q_{0}}\right)^{1-\sigma}(\beta \delta)^{\sigma} A(\beta)\right]^{1 / \sigma} \\
& -\left[1+\left(\frac{\phi w_{0}}{q_{0}}\right)^{1-\sigma}\left(\beta \delta^{2}\right)^{\sigma}\right]^{1 / \sigma}-\beta \delta x^{1-1 / \sigma}
\end{aligned}
$$

First, it is proved that $\partial f / \partial x>0$. The condition $\partial f / \partial x>0$ is equivalent to:

$$
\frac{\phi w_{0}}{\tau q_{0}}\left(x \frac{\phi w_{0}}{\tau q_{0}}+1\right)^{-1 / \sigma}\left[1+\left(\frac{\phi w_{0}}{\tau q_{0}}\right)^{1-\sigma}(\beta \delta)^{\sigma} A(\beta)\right]^{1 / \sigma}>\beta \delta x^{-1 / \sigma}
$$

which is equivalent to

$$
x>\frac{1}{\left(\frac{\phi w_{0}}{\tau q_{0}}\right)^{\sigma}(\beta \delta)^{-\sigma}+\frac{\phi w_{0}}{\tau q_{0}}[A(\beta)-1]} \equiv \Omega(\beta)
$$


From this inequality, as by assumption $x>Z(\beta)$, if $Z(\beta)>\Omega(\beta)$, the property $x>\Omega(\beta)$ will be satisfied and $\partial f / \partial x>0$.

$Z(\beta)>\Omega(\beta)$ is equivalent to:

$$
\frac{\phi w_{0}}{\tau q_{0}}\left[A(\beta)-1-(\beta \delta)^{\sigma} \tau^{1-\sigma}\right]+\left(\frac{\phi w_{0}}{\tau q_{0}}\right)^{\sigma}(\beta \delta)^{-\sigma}\left[1-\frac{1+(\beta \delta)^{\sigma} \tau^{1-\sigma}}{A(\beta)}\right]>0
$$

From the definition of $A(\beta), A(\beta)>1+(\beta \delta)^{\sigma} \tau^{1-\sigma} \Leftrightarrow 1+\beta^{\sigma-1} \delta^{\sigma} \tau^{1-\sigma}>$ $1+(\beta \delta)^{\sigma} \tau^{1-\sigma}$ which is true for $\beta<1$.

Finally the property $\partial f / \partial x>0$ is proved.

The next step is to prove that $f(Z(\beta), \beta)<0$ and $f(D(\beta), \beta)>0$. These two inequalities with the property $\partial f / \partial x>0$ will ensure the existence and uniqueness of $x$ as a function $V(\beta)$ of $\beta$.

After tedious calculations, it is possible to write $f(Z(\beta), \beta)<0$ under the form

$$
\begin{aligned}
& 1+\left(\frac{\phi w_{0}}{q_{0}}\right)^{1-\sigma}\left(\beta \delta^{2}\right)^{\sigma}\left(\frac{\beta+\delta^{\sigma} \beta^{\sigma} \tau^{1-\sigma}}{1+\delta^{\sigma} \beta^{\sigma} \tau^{1-\sigma}}\right)^{\sigma-1} \\
< & {\left[1+\left(\frac{\phi w_{0}}{q_{0}}\right)^{1-\sigma}\left(\beta \delta^{2}\right)^{\sigma}\right]^{1 / \sigma}\left[1+\left(\frac{\phi w_{0}}{q_{0}}\right)^{1-\sigma}\left(\beta \delta^{2}\right)^{\sigma}\left(\frac{\beta+\delta^{\sigma} \beta^{\sigma} \tau^{1-\sigma}}{1+\delta^{\sigma} \beta^{\sigma} \tau^{1-\sigma}}\right)^{\sigma}\right]^{1-1 / \sigma} }
\end{aligned}
$$

The following notations are introduced:

$$
\begin{aligned}
a & =\left(\frac{\phi w_{0}}{q_{0}}\right)^{1-\sigma}\left(\beta \delta^{2}\right)^{\sigma} \\
y(\beta) & =\frac{\beta+\delta^{\sigma} \beta^{\sigma} \tau^{1-\sigma}}{1+\delta^{\sigma} \beta^{\sigma} \tau^{1-\sigma}}
\end{aligned}
$$

It is possible to write the preceding inequality:

$$
\frac{\left[1+a y(\beta)^{\sigma-1}\right]^{\sigma}}{\left[1+a y(\beta)^{\sigma}\right]^{\sigma-1}}<1+a
$$

The function $y(\beta)$ increases from 0 toward 1 when $\beta$ goes from 0 to 1 .

As a function of $y$, the expression

$$
\frac{\left[1+a y^{\sigma-1}\right]^{\sigma}}{\left[1+a y^{\sigma}\right]^{\sigma-1}}
$$

is strictly increasing when $y$ goes from 0 to 1 , and is equal to $1+a$ for $y=1$. 
By these two properties, it is proved that (24) is satisfied.

The condition $f(D(\beta), \beta)>0$ can be written after some calculations

$$
\begin{aligned}
& {\left[1+\left(\frac{\phi w_{0}}{\tau q_{0}}\right)^{1-\sigma} \delta^{\sigma}\left(1+\delta^{\sigma} \beta^{\sigma} \tau^{1-\sigma}\right)\right]^{1-1 / \sigma}\left[1+\left(\frac{\phi w_{0}}{\tau q_{0}}\right)^{1-\sigma} \delta^{\sigma} \frac{\left(\beta+\delta^{\sigma} \beta^{\sigma} \tau^{1-\sigma}\right)^{\sigma}}{\left(1+\delta^{\sigma} \beta^{\sigma} \tau^{1-\sigma}\right)^{\sigma-1}}\right]^{1 / \sigma} } \\
> & 1+\left(\frac{\phi w_{0}}{\tau q_{0}}\right)^{1-\sigma} \delta^{\sigma}\left(\beta+\delta^{\sigma} \beta^{\sigma} \tau^{1-\sigma}\right)
\end{aligned}
$$

The following notations are introduced:

$$
\begin{aligned}
b & =\left(\frac{\phi w_{0}}{\tau q_{0}}\right)^{1-\sigma} \delta^{\sigma} \\
\xi & =1+\delta^{\sigma} \beta^{\sigma} \tau^{1-\sigma} \\
\theta & =\beta+\delta^{\sigma} \beta^{\sigma} \tau^{1-\sigma}
\end{aligned}
$$

By definition, $\theta \leq \xi$ with a strict inequality for $\beta<1$. It is possible to write the preceding inequality:

$$
1+b \frac{\theta^{\sigma}}{\xi^{\sigma-1}}>\frac{(1+b \theta)^{\sigma}}{(1+b \xi)^{\sigma-1}}
$$

Defining the function $g$ :

$$
g(\theta)=1+b \frac{\theta^{\sigma}}{\xi^{\sigma-1}}-\frac{(1+b \theta)^{\sigma}}{(1+b \xi)^{\sigma-1}}
$$

it is easy to check that it is strictly decreasing for $\theta \in[0, \xi]$, with $g(\xi)=0$. Therefore, it is proved that $(25)$ is satisfied, and $f(D(\beta), \beta)>0$.

\subsection{Appendix 3}

Condition (13) can be written under a condition on $w_{0}$ and $w_{1}$. The inequality $\frac{w_{2}}{w_{1}+w_{0}}>G(\beta)$ is equivalent to:

$$
w_{1}<\left(\frac{\phi \delta^{\sigma} \tau^{-\sigma} w_{2}}{q_{0}}-1\right) w_{0}+\frac{w_{2}\left(\phi w_{0}\right)^{\sigma}}{\left(\tau q_{0}\right)^{\sigma}(\beta \delta)^{\sigma}} \equiv \Gamma\left(w_{0}\right)
$$

The right-hand side member of this inequality is a function $\Gamma$ of $w_{0}$ such that: if $\frac{\phi \delta^{\sigma} \tau^{-\sigma} w_{2}}{q_{0}}>1, \Gamma$ is strictly increasing; if $\frac{\phi \delta^{\sigma} \tau^{-\sigma} w_{2}}{q_{0}}<1, \Gamma$ is U-shaped, first 
decreasing and then increasing. As $w_{1}$ cannot be negative, the negative part of $\Gamma$ does not play any role. The function $W^{G}$ is defined a

$$
W^{G}\left(w_{0}\right)=\max \left\{\Gamma\left(w_{0}\right), 0\right\}
$$

By definition, either $W^{G}\left(w_{0}\right)$ is strictly increasing, or it is first equal to 0 , and then strictly increasing.

For condition (12), the inequality $\frac{w_{2}}{w_{1}+w_{0}}>Z(\beta)$ is equivalent to:

$$
w_{1}<\left(\frac{\phi \beta^{\sigma} \delta^{\sigma} \tau^{-\sigma} w_{2}}{q_{0}}-1\right) w_{0}+\frac{w_{2}\left(\phi w_{0}\right)^{\sigma}}{\left(\tau q_{0}\right)^{\sigma} \delta^{\sigma}}\left(\frac{1+\beta^{\sigma} \delta^{\sigma} \tau^{1-\sigma}}{\beta+\beta^{\sigma} \delta^{\sigma} \tau^{1-\sigma}}\right)^{\sigma} \equiv \vartheta\left(w_{0}\right)
$$

As for the preceding example, a function $W^{Z}$ is defined as

$$
W^{Z}\left(w_{0}\right)=\max \left\{\vartheta\left(w_{0}\right), 0\right\}
$$

By definition, $W^{Z}(0)=0$, either $W^{Z}\left(w_{0}\right)$ is strictly increasing, or it is first equal to 0 , and then strictly increasing.

For condition (16), the inequality $\frac{w_{2}}{w_{1}+w_{0}}<D(\beta)$ is equivalent to:

$$
w_{1}>\left(\frac{\phi \beta^{\sigma} \delta^{\sigma} \tau^{-\sigma} w_{2}}{q_{0}}-1\right) w_{0}+\frac{w_{2}\left(\phi w_{0}\right)^{\sigma}}{\left(\tau q_{0}\right)^{\sigma} \delta^{\sigma}} \equiv \Delta\left(w_{0}\right)
$$

As for the preceding examples, a function $W^{D}$ is defined as

$$
W^{D}\left(w_{0}\right)=\max \left\{\Delta\left(w_{0}\right), 0\right\}
$$

By definition, $W^{D}(0)=0$, either $W^{Z}\left(w_{0}\right)$ is strictly increasing, or it is first equal to 0 , and then strictly increasing.

Finally condition (9) can be written:

$$
w_{1}>\frac{w_{2}\left(\phi w_{0}\right)^{\sigma}}{\left(\tau q_{0}\right)^{\sigma}(\beta \delta)^{\sigma}} \frac{\left(1+\delta^{\sigma} \beta^{\sigma} \tau^{1-\sigma}\right)^{\sigma-1}}{\left(1+\delta^{\sigma} \beta^{\sigma-1} \tau^{1-\sigma}\right)^{\sigma}}-w_{0} \equiv \Xi\left(w_{0}\right)
$$

As for the preceding examples, a function $W^{H}$ is defined as

$$
W^{H}\left(w_{0}\right)=\max \left\{\Xi\left(w_{0}\right), 0\right\}
$$

By definition, $W^{H}\left(w_{0}\right)$ is first equal to 0 , and then strictly increasing.

Lemma 4 with Appendix 2 allow to define a function $V(\beta)$ such that $\frac{w_{2}}{w_{1}+w_{0}}=V(\beta) \Leftrightarrow U^{T C}=\tilde{U}^{T C}$. This function $V(\beta)$ depends on different 
parameters of the model including $w_{0}$, but does not depend on $w_{1}$. Therefore, it is clear that it can be expressed under the form:

$$
w_{1}=\frac{w_{2}}{V(\beta)}-w_{0}
$$

A function $W^{V}$ is defined as:

$$
W^{V}\left(w_{0}\right)=\max \left\{\frac{w_{2}}{V(\beta)}-w_{0}, 0\right\}
$$

As $Z(\beta)<V(\beta)<D(\beta)$, it implies that: $W^{D}\left(w_{0}\right)<W^{V}\left(w_{0}\right)<W^{Z}\left(w_{0}\right)$.

To find how $W^{V}\left(w_{0}\right)$ evolves with $w_{0}$, it is useful to come back to the definition. When $w_{1}=W^{V}\left(w_{0}\right)$ is positive, the function is implicitly defined by the relation $U^{T C}-\tilde{U}^{T C}=0$. The derivative is implicitly given by:

$$
\frac{d W^{V}\left(w_{0}\right)}{d w_{0}}=-\frac{\frac{\partial\left(U^{T C}-\tilde{U}^{T C}\right)}{\partial w_{0}}}{\frac{\partial\left(U^{T C}-\tilde{U}^{T C}\right)}{\partial w_{1}}}
$$

If $\frac{\partial\left(U^{T C}-\tilde{U}^{T C}\right)}{\partial w_{0}} \neq 0$, it will prove that $W^{V}\left(w_{0}\right)$ is monotonic. As $W^{D}\left(w_{0}\right)<$ $W^{V}\left(w_{0}\right)<W^{Z}\left(w_{0}\right)$, with $W^{D}\left(w_{0}\right)$ and $W^{Z}\left(w_{0}\right)$ two increasing functions tending to $+\infty$ when $w_{0} \rightarrow+\infty$, the only possibility will be that $W^{V}\left(w_{0}\right)$ is monotonically increasing.

It is possible to prove that $\frac{\partial\left(U^{T C}-\tilde{U}^{T C}\right)}{\partial w_{0}}>0 . U^{T C}$ is the maximum value of self 1 's objective function when $q^{T C}(m)>0 . \tilde{U}^{T C}$ is the maximum value of self 1's objective function when $q^{T C}(m)=0$. The derivatives can be obtained using the envelope theorem:

$$
\begin{aligned}
\frac{\partial U^{T C}}{\partial w_{0}} & =\left(1-\phi m^{T C}\right)\left[w_{1}+w_{0}\left(1-\phi m^{T C}\right)\right]^{-\frac{1}{\sigma}} \\
\frac{\partial \tilde{U}^{T C}}{\partial w_{0}} & =\left(1-\phi \tilde{m}^{T C}\right)\left[w_{1}+w_{0}\left(1-\phi \tilde{m}^{T C}\right)\right]^{-\frac{1}{\sigma}}
\end{aligned}
$$

As $\sigma>1$, the function $x\left[w_{1}+w_{0} x\right]^{-\frac{1}{\sigma}}$ is an increasing function of $x$, as its logarithmic derivative is

$$
\frac{\sigma w_{1}+(\sigma-1) w_{0} x}{x\left(w_{1}+w_{0} x\right) \sigma}
$$

Consequently, the function $(1-\phi m)\left[w_{1}+w_{0}(1-\phi m)\right]^{-\frac{1}{\sigma}}$ is a decreasing function of $m$. As $\tilde{m}^{T C}>m^{T C}$, it is obtained that,

$$
\frac{\partial U^{T C}}{\partial w_{0}}>\frac{\partial \tilde{U}^{T C}}{\partial w_{0}}
$$


which implies that the function $W^{V}\left(w_{0}\right)$ is increasing.

\subsection{Appendix 4}

From (10), $m^{C}$ can be written:

$$
m^{C}=\frac{x(\tau)\left(w_{1}+w_{0}\right)-\frac{w_{2}}{\tau q_{0}}}{1+\phi w_{0} x(\tau)}
$$

with $x(\tau) \equiv(\beta \delta)^{\sigma} q_{0}^{\sigma-1}\left(\phi w_{0}\right)^{-\sigma}\left(\tau^{\sigma-1}+\delta^{s}\right)$. Taking the derivative of $m^{C}$ with respect to $\tau$, it is obtained that the sign of this derivative is the sign of the expression:

$$
\begin{aligned}
& {\left[x^{\prime}(\tau)\left(w_{1}+w_{0}\right)+\frac{w_{2}}{\tau^{2} q_{0}}\right]\left[1+\phi w_{0} x(\tau)\right]-\left[x(\tau)\left(w_{1}+w_{0}\right)-\frac{w_{2}}{\tau q_{0}}\right]\left[\phi w_{0} x^{\prime}(\tau)\right] } \\
= & x^{\prime}(\tau)\left[\left(w_{1}+w_{0}\right)+\phi w_{0} \frac{w_{2}}{\tau q_{0}}\right]+\frac{w_{2}}{\tau^{2} q_{0}}\left[1+\phi w_{0} x(\tau)\right]>0
\end{aligned}
$$

Thus, $m^{C}$ is an increasing function of $\tau$.

From (5), the quality level $q$ is such that:

$$
q_{0} \tau^{\sigma}+q\left(\tau^{\sigma}+\delta^{\sigma} \tau\right)=\delta^{\sigma} \frac{w_{2}}{m^{C}}
$$

If $\tau$ increases, as $m^{C}$ increases, $q$ must decrease.

From (8), the time-consistent solution can be written:

$$
m^{T C}=\frac{y(\tau)\left(w_{1}+w_{0}\right)-\frac{w_{2}}{\tau q_{0}}}{1+\phi w_{0} y(\tau)}
$$

with $y(\tau) \equiv(\beta \delta)^{\sigma} q_{0}^{\sigma-1}\left(\phi w_{0}\right)^{-\sigma} \tau^{\sigma-1} A(\beta)$. If $y^{\prime}(\tau)>0$, it is known from the preceding calculation that $m^{T C}$ increases with $\tau$, as the sign of $\partial m^{T C} / \partial \tau$ is given by

$$
y^{\prime}(\tau)\left[\left(w_{1}+w_{0}\right)+\phi w_{0} \frac{w_{2}}{\tau q_{0}}\right]+\frac{w_{2}}{\tau^{2} q_{0}}\left[1+\phi w_{0} y(\tau)\right]>0
$$

Therefore, it remains to check if $\tau^{\sigma-1} A(\beta)$ increases with $\tau$. After some calculations, it is obtained that

$$
\frac{\partial y(\tau) / \partial \tau}{y(\tau)}=\frac{d \ln \left[\tau^{\sigma-1} A(\beta)\right]}{d \tau}=(\sigma-1) \frac{1-[\sigma(1-\beta)-1] \delta^{\sigma} \beta^{\sigma-1} \tau^{1-\sigma}}{\tau\left(1+\delta^{\sigma} \beta^{\sigma-1} \tau^{1-\sigma}\right)\left(1+\delta^{\sigma} \beta^{\sigma} \tau^{1-\sigma}\right)}
$$


If $\sigma(1-\beta)<1, y^{\prime}(\tau)>0$ and $m^{T C}$ increases with $\tau$. If $\sigma(1-\beta)>1$, it is not possible to achieve a general conclusion. For $\tau$ small enough $(\tau \rightarrow 0)$, it is possible to show from (26) that

$$
y^{\prime}(\tau)\left[\left(w_{1}+w_{0}\right)+\phi w_{0} \frac{w_{2}}{\tau q_{0}}\right]+\frac{w_{2}}{\tau^{2} q_{0}}\left[1+\phi w_{0} y(\tau)\right] \underset{\tau \rightarrow 0}{\sim} \tau^{-2}\left(a-b \tau^{\sigma-1}\right)
$$

with $a$ and $b$ two positive constant parameters. Therefore, $m^{T C}$ increases with $\tau$ when $\tau$ is small.

Assuming that $m^{T C}$ increases with $\tau$, from (4), the quality level $q$ is such that:

$$
q_{0} \tau^{\sigma}+q\left(\tau^{\sigma}+(\beta \delta)^{\sigma} \tau\right)=(\beta \delta)^{\sigma} \frac{w_{2}}{m^{T C}}
$$

If $\tau$ increases, as $m^{T C}$ increases, $q$ must decrease.

Considering now $q^{T C}\left(m^{T C}\right)$, Appendix 1 has shown that

$$
q^{T C}\left(m^{T C}\right)+q_{o}=\frac{(\beta \delta / \tau)^{\sigma}\left(\frac{w_{2}}{m^{T C}}+\tau q_{0}\right)}{1+(\beta \delta)^{\sigma} \tau^{1-\sigma}}
$$

or

$q^{T C}\left(m^{T C}\right)+q_{o}=\frac{(\beta \delta)^{\sigma}\left[\tau q_{0}\left(w_{1}+w_{0}\right)+w_{2} \phi w_{0}\right]}{\tau^{\sigma}+(\beta \delta)^{\sigma} \tau} \frac{(\beta \delta)^{\sigma} A(\beta) B}{(\beta \delta)^{\sigma} A(\beta) B\left(w_{1}+w_{0}\right)-w_{2}}$

It is easy to check that the first term

$$
\frac{(\beta \delta)^{\sigma}\left[\tau q_{0}\left(w_{1}+w_{0}\right)+w_{2} \phi w_{0}\right]}{\tau^{\sigma}+(\beta \delta)^{\sigma} \tau}=\frac{(\beta \delta)^{\sigma}\left[q_{0}\left(w_{1}+w_{0}\right)+\frac{w_{2} \phi w_{0}}{\tau}\right]}{\tau^{\sigma-1}+(\beta \delta)^{\sigma}}
$$

is a decreasing function of $\tau$ as $\sigma>1$.

Defining $z(\tau)=(\beta \delta)^{\sigma} A(\beta) B$, the second term can be written

$$
\frac{z(\tau)}{z(\tau)\left(w_{1}+w_{0}\right)-w_{2}}
$$

This is a decreasing function with respect to $z(\tau)$. Therefore, if $z(\tau)$ increases with $\tau$, it will be obtained that $q^{T C}\left(m^{T C}\right)$ is a decreasing function of $\tau$.

$z(\tau)$ can be written:

$$
z(\tau)=(\beta \delta)^{\sigma}\left(\frac{q_{0}}{\phi w_{0}}\right)^{\sigma} \frac{\left(\tau^{\sigma}+\delta^{\sigma} \beta^{\sigma-1} \tau\right)^{\sigma}}{\left(\tau^{\sigma}+\delta^{\sigma} \beta^{\sigma} \tau\right)^{\sigma-1}}
$$


The sign of $z^{\prime}(\tau) / z(\tau)$ is given by the sign of:

$$
\tau^{2 \sigma-1}+\tau^{\sigma} \delta^{\sigma} \beta^{\sigma-1}\left[-\sigma^{2}(1-\beta)+(2-\beta) \sigma+\beta\right]+\delta^{2 \sigma} \beta^{2 \sigma-1}
$$

A sufficient condition to have $z^{\prime}(\tau)>0$ is that $-\sigma^{2}(1-\beta)+(2-\beta) \sigma+\beta>0$. Considering this second degree equation in $\sigma$, the property $z^{\prime}(\tau)>0$ will hold if

$$
\sigma<\bar{\sigma} \equiv \frac{2-\beta+\sqrt{4-3 \beta^{2}}}{2(1-\beta)}
$$

It is easy to check that $\bar{\sigma}>1 /(1-\beta)$ as it is equivalent to $\beta<1$.

\subsection{Appendix 5}

This appendix proves that $V(\beta)$ increases with $\tau$. This function has been defined in Appendix 2 as the solution $x$ implicitly defined by the equation: $f(x, \beta)=0$ with $x=\frac{w_{2}}{w_{1}+w_{0}}$ and

$$
f(x, \beta)=\frac{\sigma-1}{\sigma}\left(U^{T C}-\tilde{U}^{T C}\right) \frac{1}{\left(w_{1}+w_{0}\right)^{1-1 / \sigma}}
$$

From this definition, it appears that

$$
\frac{\partial V(\beta)}{\partial \tau}=-\frac{\frac{\partial f}{\partial \tau}}{\frac{\partial f}{\partial x}}
$$

In Appendix 2, it was shown that $\frac{\partial f}{\partial x}>0$. It remains to prove that $\frac{\partial f}{\partial \tau}<0$, which is equivalent to prove that: $\frac{\partial\left(U^{T C}-\tilde{U}^{T C}\right)}{\partial \tau}<0 . U^{T C}$ is the maximum value of self 1's objective function when $q^{T C}(m)>0 . \tilde{U}^{T C}$ is the maximum value of self 1 's objective function when $q^{T C}(m)=0$. The derivatives can be obtained using the envelope theorem:

$$
\begin{aligned}
& \left.\frac{\partial U^{T C}}{\partial w_{0}}=-m^{T C} q^{T C} \beta \delta\left[w_{2}-\tau m^{T C} q^{T C}\right)\right]^{-\frac{1}{\sigma}} \\
& \frac{\partial \tilde{U}^{T C}}{\partial w_{0}}=0
\end{aligned}
$$

Therefore, $\frac{\partial\left(U^{T C}-\tilde{U}^{T C}\right)}{\partial \tau}<0$ and $V(\beta)$ is an increasing function of $\tau$. 